\title{
Effective models of two-flavor QCD: Phase diagram and the bare quark mass
}

\section{Topi Kähärä*}

University of Jyväskylä and Helsinki Institute of Physics, Finland

E-mail: topi.kahara@ jyu.fi

\section{Kimmo Tuominen}

University of Jyväskylä and Helsinki Institute of Physics, Finland

E-mail: kimmo.tuominen@phys.jyu.fi

We study effective models of chiral fields and Polyakov loop expected to describe the dynamics responsible for the phase structure of two-flavor QCD at finite temperature and density. We consider the chiral sector described either using linear sigma model or Nambu-Jona-Lasinio model, study the phase diagram and determine the location of the critical point as a function of the explicit chiral symmetry breaking (i.e. the bare quark mass).

The many faces of $Q C D$

November 1-5, 2010

Gent, Belgium

\footnotetext{
* Speaker.
} 


\section{Introduction}

Hadronic matter undergoes a phase transition from hadronic matter into a partonic matter at high temperatures or densities. To predict the equation of state, study the existence of possible critical point(s) in the $(T, \mu)$ - phase diagram and the properties of the phase transitions presents a theoretical challenge on studies based on the fundamental theory of strong interactions, QCD. To obtain some insight into the QCD dynamics of quarks in the nonperturbative domains, models like the Nambu-Jona-Lasinio (NJL) model have been developed. These models are based on the chiral symmetry of light quarks.

Based on generic effective theory methods, identification of relevant degrees of freedom and symmetries, effective models able to account for the two most important features of QCD, deconfinement and chiral symmetry breaking, the effective models featuring both chiral and Polyakov loop degrees of freedom have been developed and studied [1,2]. The chiral symmetry is effectively represented by a NJL model or linear sigma model (LSM), while the $Z_{3}$ symmetry relevant for deconfinement in pure gauge theory is described by a mean field potential for Polyakov loop. The key role is played by interactions coupling these two sectors. In the limit of light quarks the dynamics are driven by the chiral degrees of freedom and the decrease in the chiral condensate as the temperature is increased results in increase of the Polyakov loop which in turn results in deconfinement and explains the coincidence of these two seemingly unrelated features to a single phase transition $[3,4,5,6,7]$. On the other hand, if the current quark masses are taken large, chiral symmetry broken explicitly, the dynamics is close to that of pure gauge theory, i.e. dominated by the Polyakov loop. Nevertheless, at deconfimenent the interactions now lead to decrease in the chiral condensate and the two transitions again coincide. In real QCD approximate chiral symmetry is typically expected to play the dominant role.

\section{The models}

We consider side by side the Nambu-Jona-Lasinio (NJL) model and the linear sigma model (LSM) both extended with a lattice fitted potential for the Polyakov loop and an interaction between the Polyakov loop and the chiral sector. The resulting models are abbreviated PNJL and PLSM, respectively. The Lagrangians of the models are of the form

$$
\mathscr{L}=\mathscr{L}_{\text {chiral }}+\mathscr{L}_{\text {polyakov }}+\mathscr{L}_{\text {interaction }}
$$

and the only difference in the models is in the chiral sector provided by the NJL or LSM. The linear sigma model (LSM) consist of the sigma meson and the pions with their mutual interactions, and interactions with quarks. The NJL model on the other hand describes only quarks with an effective four-fermion interaction. Both models break the chiral symmetry explicitly. For the exact form of the chiral Lagrangians, see [8].

The Polyakov loop is included to both models through the mean field potential

$$
U_{\ell} \equiv U\left(\ell, \ell^{*}, T\right)=T^{4}\left(-\frac{b_{2}(T)}{2}|\ell|^{2}-\frac{b_{3}}{6}\left(\ell^{3}+\ell^{* 3}\right)+\frac{b_{4}}{4}\left(|\ell|^{2}\right)^{2}\right),
$$


where

$$
b_{2}(T)=a_{0}+a_{1}\left(\frac{T_{0}}{T}\right)+a_{2}\left(\frac{T_{0}}{T}\right)^{2}+a_{3}\left(\frac{T_{0}}{T}\right)^{3},
$$

and the constants $a_{i}, b_{i}$ are fixed to reproduce pure gauge theory thermodynamics with phase transition at $T_{0}=270 \mathrm{MeV}$; We adopt the values determined in [4]. Later we will introduce a $\mu$ dependence in the potential through the $T_{0}$ parameter as done in [9].

This study is done in the mean field approximation. For details, see [8]. Here we simply state the grand potential

$$
\Omega=U_{\text {chiral }}+U_{\ell}+\Omega_{\bar{q} q}
$$

where the chiral potentials are

$$
\begin{aligned}
U_{\text {chiral }} & =\frac{\lambda^{2}}{4}\left(\left(\frac{M}{g}\right)^{2}-v^{2}\right)^{2}-\frac{H M}{g}, \text { for LSM } \\
U_{\text {chiral }} & =\frac{\left(m_{q}-M\right)^{2}}{2 G}, \text { for NJL. }
\end{aligned}
$$

The constituent masses $M$ are related to the $\bar{q} q$ and $\sigma$ expectation values throught the relations $M=m_{q}-G\langle\bar{q} q\rangle$ in NJL and $M=g\langle\sigma\rangle$ in LSM. The final term in (2.4) includes the interaction between the chiral and Polyakov loop $\ell$ and is

$$
\begin{aligned}
\Omega_{\bar{q} q}= & -2 N_{f} T \int \frac{d^{3} p}{(2 \pi)^{3}}\left(\ln \left[1+3\left(\ell+\ell^{*} e^{-(E-\mu) / T}\right) e^{-(E-\mu) / T}+e^{-3(E-\mu) / T}\right]\right. \\
& \left.+\ln \left[1+3\left(\ell^{*}+\ell e^{-(E+\mu) / T}\right) e^{-(E+\mu) / T}+e^{-3(E+\mu) / T}\right]\right),
\end{aligned}
$$

where $E=\sqrt{\vec{p}^{2}+M^{2}}$ and $M$ is the constituent mass of the model in question as defined above. The interaction potential $\Omega_{\bar{q} q}$ includes also a vacuum term omitted from equation (2.7)

$$
\Omega_{\bar{q} q}^{v a c}=-6 N_{f} \int \frac{d^{3} p}{(2 \pi)^{3}} E \theta\left(\Lambda^{2}-|\vec{p}|^{2}\right) .
$$

This term is included as it is in the PNJL model and regulated by the cutoff $\Lambda$. In the PLSM case we instead use the renormalized form from [11]

$$
\Omega_{\bar{q} q}^{r e g}=-\frac{6}{8 \pi^{2}} M^{4} \ln \left(\frac{M}{\Lambda}\right),
$$

where the renormalization scale $\Lambda$ is chosen to be the same as the cutoff in the PNJL case.

Note that in our works $[8,10]$ we neglected the fermion vacuum term in the PLSM case. In [11] it was observed that the inclusion of this vacuum term is needed to maintain the transition second order at the chiral limit as required by the $O(4)$ universality class. The influence of the fermion vacuum term on the location of the critical point has also been suggested or studied in e.g. $[12,13,14,15]$. In this work the main results for PLSM contain the vacuum term contribution.

At the physical pion mass the parameters of the models are fixed by the physical vacuum properties. The parameter values for both models at the physical pion mass and further details of 
the parameter fitting can be found in [16]. To explore the parameter space away from the physical pion mass we need a consistent way of setting the model parameters. In the PNJL model this is easy since the bare quark mass is a direct input parameter of the model and is directly responsible for the explicit chiral symmetry breaking. The PLSM case is a little more involved since the pion and sigma masses as well as the pion decay constant are independent input parameters which, however, should be connected with each other. To overcome this problem we use a lattice based parametrisition of the PLSM parameters that relates them with the bare quark mass $m_{q}$ making their consistent tuning and comparison with the PNJL model possible. This scheme was introduced in [8] and slightly improved in [16]. We will not discuss it's details here. However, in Figure 1 the pion mass and decay constant are shown as functions of the bare quark mass, where the PLSM results correspond to the lattice fit.

The thermodynamics of the models are determined by solving the equations of motion for the order parameters,

$$
\frac{\partial \Omega}{\partial M}=0, \frac{\partial \Omega}{\partial \ell}=0, \frac{\partial \Omega}{\partial \ell^{*}}=0,
$$

and then the pressure is given by evaluating the potential on the minimum, $p=-\Omega(T, \mu)$. We have chosen the constituent mass $M$ as a basic variable since this most conveniently allows us to discuss both models simultaneously. It is also straightforward to write the results in terms of the condensates $\langle\sigma\rangle$ and $\langle\bar{q} q\rangle$ since these are linearly related to $M$ in each case. At finite chemical potential the mean field potential $\Omega$ is complex due to the Polyakov loops and minimizing such a potential is meaningless. A simple way to overcome this problem is to treat the Polyakov loop parameters $\ell$ and $\ell^{*}$ as independent real variables, which will be a sufficient approximation for our current analysis. Inaccuracies of this treatment as well as improved methods have been discussed for example in $[5,17]$.
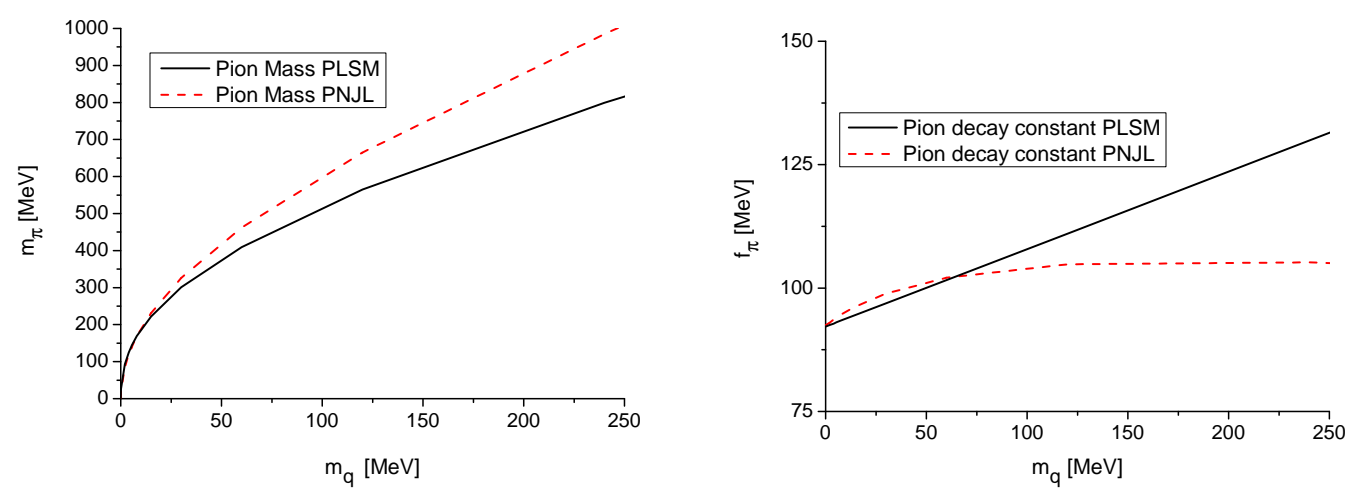

Figure 1: Pion mass $m_{\pi}$ and pion decay constants $f_{\pi}$ as functions of the bare quark mass $m_{q}$ in the PLSM and PNJL models.

\section{Results}

In the chiral models extended with Polyakov loop there are, by contruction, two transitions: the chiral transition associated with the spontaneus breaking of chiral symmetry and the deconfine- 
ment transition described by the Polyakov loop. The transitions can be studied through their order parameters, the constituent mass $M$ and the Polyakov loop $\ell$. One can assign to both transitions their own pseudo-critical temperatures. The definition of such pseudo-critical temperature, however, is vague especially in the regions where the transition is a crossover and the order parameter shifts continuously. Since this is the case over large portion of the $(T, \mu)-$ plane, in this work we primarily define the transition temperature as the temperature at which the temperature derivative of the order parameter has a maximum. Even this definition has some problems, since in some cases the derivative has several local maxima indicating rapid changes at several different temperatures. The critical temperature is identified with the maximum at which the change in the absolute value of the corresponding order parameter is largest. Alternatively one could use the susceptibilities to define the critical temperature.

\subsection{The chiral phase diagram and the critical point}

The chiral transition can be determined by finding the temperature corresponding to the fastest change in the constituent quark mass $M$ at fixed chemical potential $\mu$ (or vice versa). This transition temperature corresponds in most cases to the temperature at which the constituent mass drops below $50 \%$ of its vacuum value, only at large $m_{q}$ and $\mu$ does the fastest change occur at a different temperature than the one where the decrease in the absolute value of the constituent mass takes place. Figure 2 shows the chiral transition lines in the $(T, \mu)$-plane for different quark masses for both models; also the critical points are shown. The critical points indicate the points where a line of first order (discontinuous) transitions ends and turns into a crossover (continuous).

As seen in Figure 2, the qualitative features of the phase diagrams in the two models are very similar: As the quark mass rises, the area under the transition line expands and the critical point moves towards larger $\mu$. The quantitative difference in the transition temperature between the models is below $15 \%$ for the shown quark masses. However, the critical points appear to behave differently in the models especially at large values of $m_{q}$.

In PLSM the critical point is not present for the four largest quark masses in Figure 2 and the transition is a crossover throughout the $(T, \mu)$-plane. For the physical quark mass our result is consistent with [12] and [14], although both exhibit higher temperatures for the critical point. We also studied PLSM without the vacuum term (2.8) and found that in that case the critical point disappears also at the low $m_{q}$ region, but now from the other end of the transition line, leaving the transition first order across the whole $(T, \mu)$-plane. A similar result for a three flavour linear sigma model has been obtained in [18], where the vacuum term has also been omitted. We note that in PLSM with the fermion vacuum term the value of $m_{\pi}$ above which there is no critical point is similar to [19].

In the PNJL case the critical points are present for all quark masses shown in Figure 2 and the transitions near zero chemical potential remain crossovers. In high $\mu$ region the temperature of the critical point starts to rise again when going to larger quark masses and does not disappear as in the PLSM case. A similar effect has been observed in [17], where a saturation of the critical point temperature was mentioned and attributed to a diquark dominated phase. Since our work does not include diquark degrees of freedom, we conclude that the behaviour of the critical point at large quark masses is a more generic feature of the model. Also for two flavor QCD, a model calculation [20] using the Cornwall-Jackiw-Tomboulis effective potential in the improved-ladder approxima- 
tion exhibits very similar $m_{q}$ dependence of the critical point: the minimum $T_{c}(\mu)$ appears at nearly the same values of $m_{q}$ in [20] as in our analysis of the PNJL model, although the temperatures of the critical points vary clearly more in the PNJL model.

It has been suggested in beyond mean field calculations e.g. [19] and [21], that there might be, especially at small quark masses, multiple critical points in the $(T, \mu)$-plane. We, however, found no evidence in either model to suggest that this is the case. In our previous work [8] we noted that at large quark masses the PLSM transition was first order at $\mu=0$ giving some credence to the idea of multiple critical points at large quark masses. As mentioned in the previous paragraph and evident from Figure 2, this is not the case in our present work and the first order transition at $\mu=0$ observed in [8] was caused by the overestimation of the nucleon mass $M_{N}$ at large pion masses and the resulting overestimation of the coupling $g$. In this work with a more precise formula for the nucleon mass as well as the inclusion of the vacuum term (2.8) in the potential, the transition is a crossover for all quark masses.
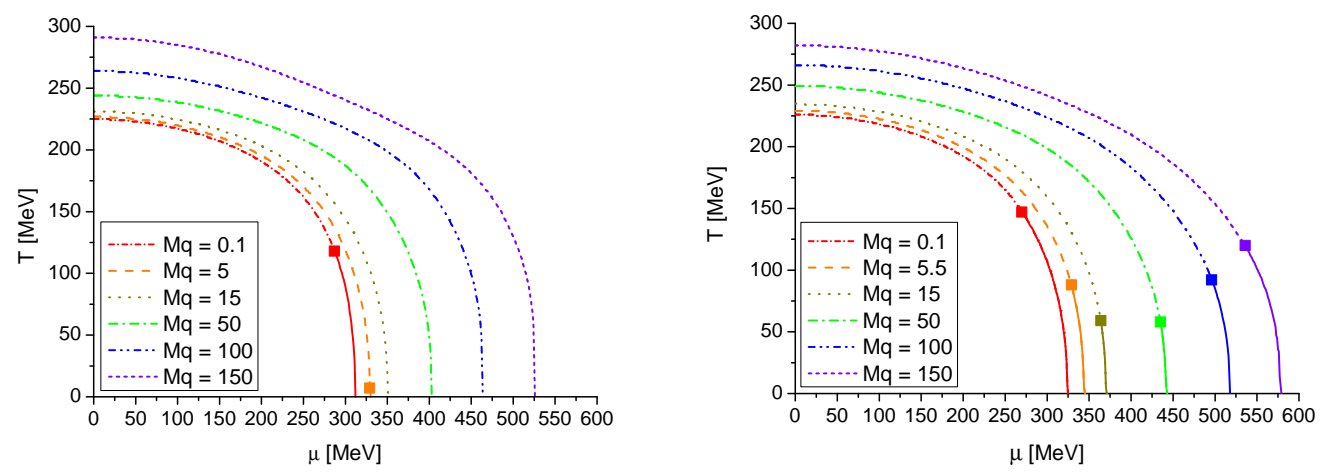

Figure 2: The chiral phase diagrams of the models for several quark masses. The solid curves indicate a first order (discontinuous) transition with the critical endpoints marked by squares. Left: PLSM Right: PNJL

\subsection{The deconfinement transition}

The deconfinement of the system is quantified by the Polyakov loop order parameter $\ell$ and its conjugate $\ell^{*}$, which we treat as independent real variables. As with the chiral transition the transition temperatures could be determined locating the maxima of the temperature derivatives. However, as noted in our previous works $[10,8,16]$ there are some problems associated with this choice. To avoid these problems we use the absolute value of the Polyakov loop to define the transition and since the Polyakov loop order parameters $\ell$ and $\ell^{*}$ obtain, with our choice of potential, values roughly from 0 to 1 , we choose the values $\ell=1 / 2$ and $\ell^{*}=1 / 2$ as indicators when the system turns from a mostly confined state to a mostly deconfined state, bearing in mind that the transition is a crossover. To further simplify the analysis and readability of the figures we will, since $\ell$ and $\ell^{*}$ do not coincide at finite $\mu$, define single deconfinement transition temperature as the average of the transition temperatures determined by $\ell=1 / 2$ and $\ell^{*}=1 / 2$.

In Figures 3 and 4 the averaged deconfinement transition lines are shown for the physical quark mass $m_{q} \approx 5 \mathrm{MeV}$ and a larger quark mass $m_{q}=50 \mathrm{MeV}$ along with the corresponding 
chiral transitions. The first observation is that, defined this way, the deconfinement transition is independent of the chiral model and also of the amount of explicit chiral symmetry breaking i.e. the quark mass. This means that tuning the Polyakov potential, so that one obtains a coincidence of the deconfining and chiral transitions for one chiral model and a specific quark mass, will not give the same outcome in other cases. Furthermore, the deconfinement transition line, $T_{c, \mathrm{dec}}(\mu)$, depends only very weakly on $\mu$. This means that deconfinement and chiral restoration can be made to coincide only at $\mu=0$. However, on the basis of symmetries one does not expect deconfinement and chiral restoration to become independent at finite $\mu$. Hence one is led to study possible $\mu$ dependence in the Polyakov loop potential.
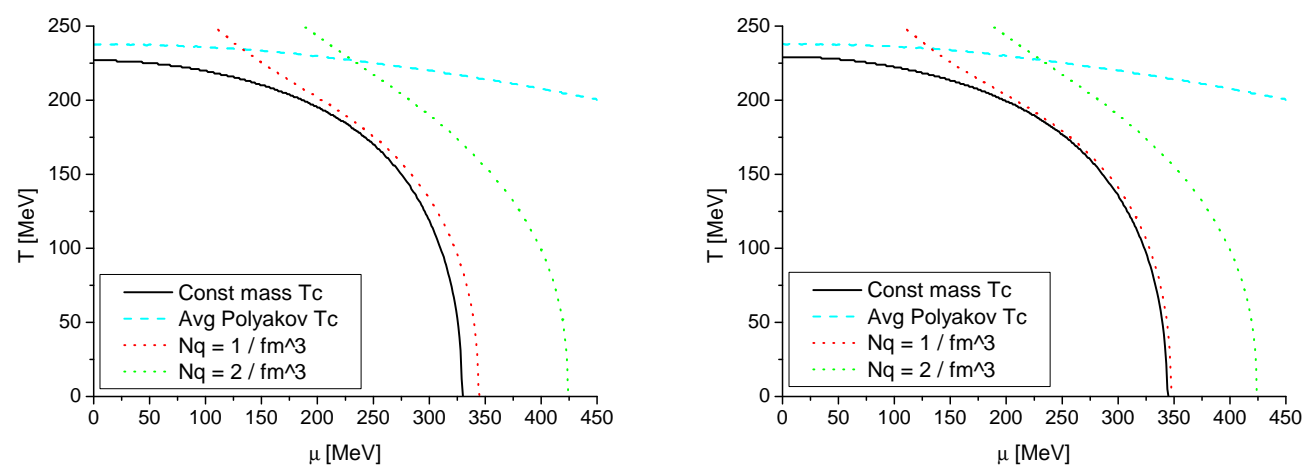

Figure 3: The phase diagrams of the models at the physical point $m_{q} \approx 5 \mathrm{MeV}$ with no explicit $\mu$ dependence in the Polyakov potential. The solid line is the chiral transition and the dashed line the deconfinement transition. The dotted lines correspond to quark number densities of $1 \mathrm{fm}^{-3}$ and $2 \mathrm{fm}^{-3}$. Left: PLSM Right: PNJL.
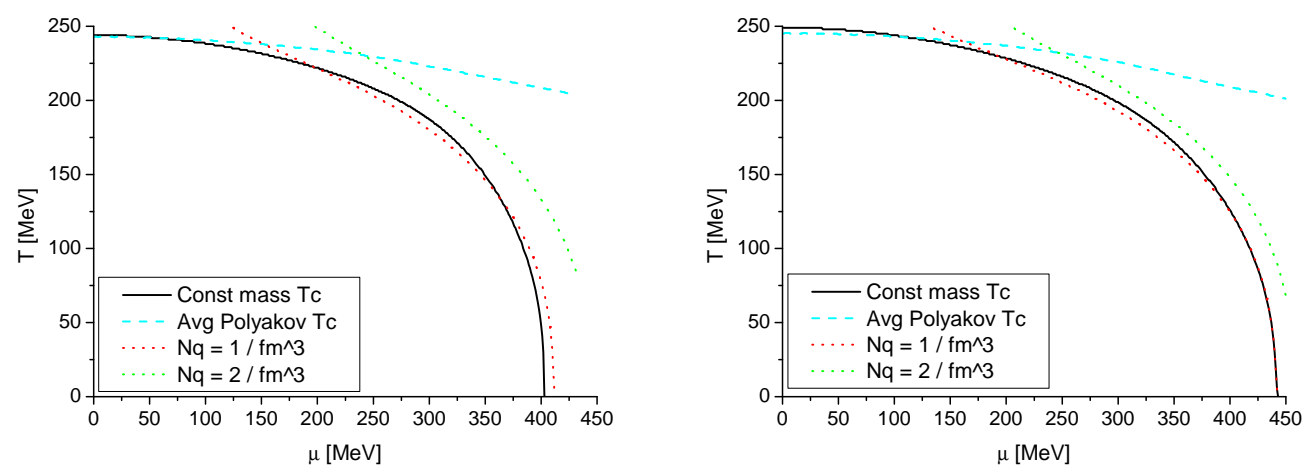

Figure 4: The phase diagrams of the models at $m_{q}=50 \mathrm{MeV}$ with no explicit $\mu$-dependence in the Polyakov potential. The solid line is the chiral transition and the dashed line the deconfinement transition. The dotted lines correspond to quark number densities of $1 \mathrm{fm}^{-3}$ and $2 \mathrm{fm}^{-3}$. Left: PLSM Right: PNJL.

To illustrate the effects of $\mu$-dependence of the Polyakov potential on the deconfinement transition, we adopt the formulation from [9] with the following modifications: In [9] the $\mu-$ dependence of the Polyakov potential was through the critical temperature $T_{0}$, which could be 
described by the following parametrization

$$
T_{0}(\mu)=T_{\tau} e^{-1 /\left(\alpha_{0} b(\mu)\right)},
$$

with the coefficient $b(\mu)$ depending on the number of colors, massless flavors and the chemical potential. The parameters $\alpha_{0}$ and $T_{\tau}$ are fixed to reproduce the $N_{f}=0$ lattice results. Details and parameter values can be found in [16].

The phase diagrams obtained using the $\mu$-dependent Polyakov potential are shown in Figures 5 and 6 . The deconfinement transition now appears very different: The main new feature is that the deconfinement temperature follows the chiral restoration critical temperature more closely. As lattice indicates [22], the deconfinement phase transition and chiral symmetry restoration occur at comparable temperatures at $\mu=0$. If this feature was seen to persist also at finite $\mu$, it would lend strong support for $\mu$-dependent Polyakov loop potential.
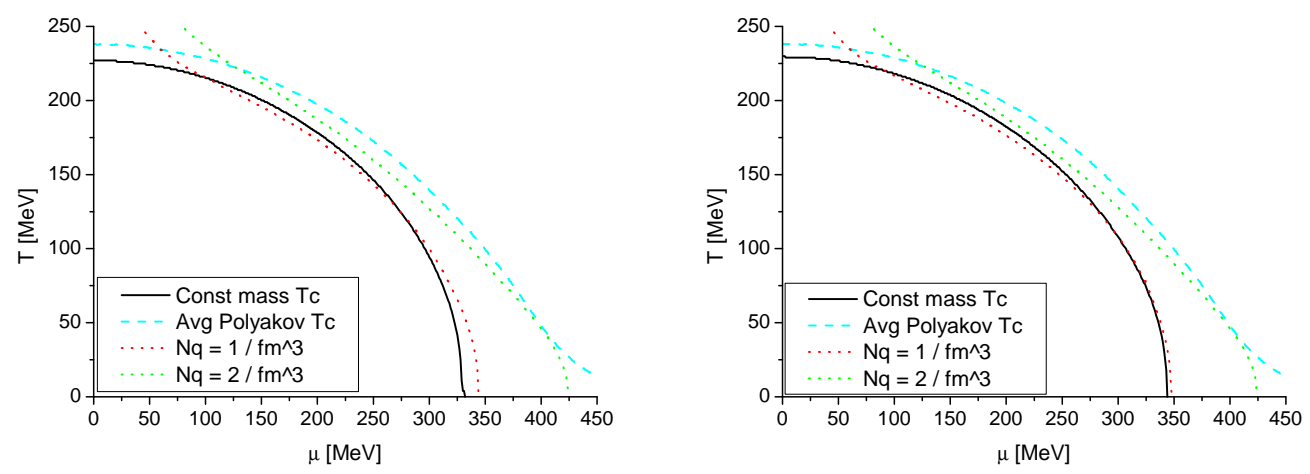

Figure 5: The phase diagrams of the models at the physical point $m_{q} \approx 5 \mathrm{MeV}$ with a $\mu$-dependent Polyakov potential. The solid line is the chiral transition and the dashed line the deconfinement transition. The dotted lines correspond to quark number densities of $1 \mathrm{fm}^{-3}$ and $2 \mathrm{fm}^{-3}$. Left: PLSM Right: PNJL (Color online)
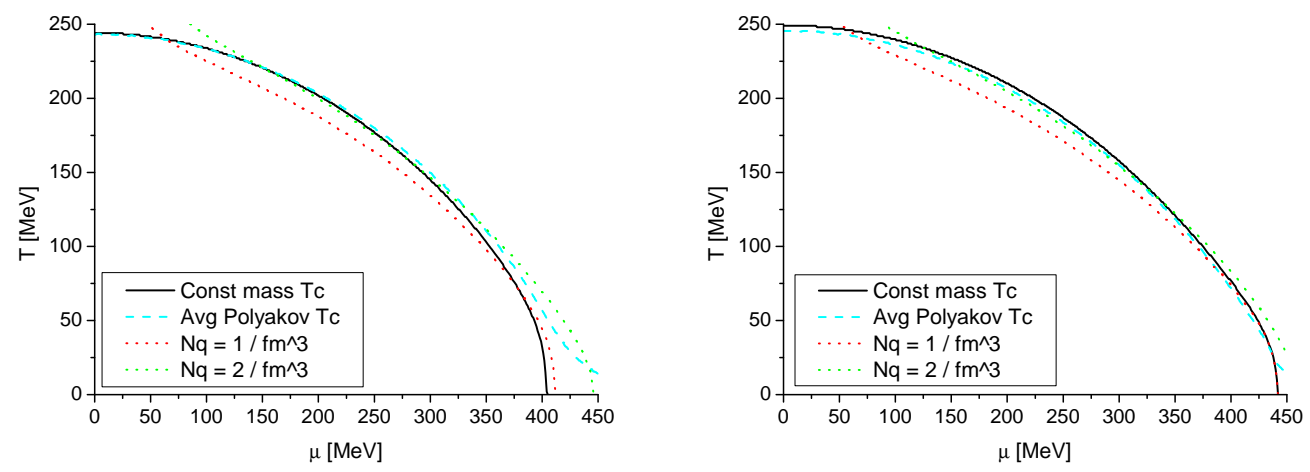

Figure 6: The phase diagrams of the models at $m_{q}=50 \mathrm{MeV}$ with a $\mu$-dependent Polyakov potential. The solid line is the chiral transition and the dashed line the deconfinement transition. The dotted lines correspond to quark number densities of $1 \mathrm{fm}^{-3}$ and $2 \mathrm{fm}^{-3}$. Left: PLSM Right: PNJL (Color online) 


\section{Conclusions}

We have considered the $(T, \mu)$ - phase diagram of two-flavor QCD in effective models which take into account both chiral degrees of freedom relevant for the restoration of the chiral symmetry and Polyakov loop relevant for deconfinement. Earlier these studies have been performed by constraining these models to reproduce the physical vacuum, in the two flavor case essentially determined by the bare quark mass $m_{q}=m_{u}=m_{d}$ (or alternatively by the pion mass $m_{\pi}$ ). We have relaxed this assumption and treated $m_{q}$ as a free parameter of the model in order to study how the explicit chiral breaking manifests in the thermodynamics.

We considered, side by side, two different models PNJL and PLSM which differ by the choice of the effective realization for the chiral sector. In earlier studies these two models have been shown to lead to qualitatively similar results for the thermodynamics both at finite temperature and density; the main quantitative difference has been shown to be in the location of the critical point in the $(T, \mu)$ - phase diagram. In this paper we have shown that, as a function of $m_{q}$, a qualitative difference arises: while in PNJL model the critical point exists for any $m_{q}$, in PLSM model the critical point exists only for a range of values of $m_{q}$. In both models we find, for any $m_{q}$, at most one critical point in contrast to the results in [19] and [21], where multiple critical points were observed for LSM model at non-physical values of the pion mass.

Concerning the deconfinement transition in these models, we conclude that in order to achieve coincidence of the chiral and deconfinement transitions thoughout the $(T, \mu)$ - plane, one has to include a $\mu$-dependence to the Polyakov sector. We also find that the renormalization group motivated form for this dependence introduced in [9] may lead to a total coincidence of the two transitions across the whole $(T, \mu)$ - plane.

\section{Acknowledgments}

The financial support for T.K. from the Väisälä foundation is gratefully acknowledged.

\section{References}

[1] A. Mocsy, F. Sannino and K. Tuominen, Phys. Rev. Lett. 92, 182302 (2004) [arXiv:hep-ph/0308135]; A. Mocsy, F. Sannino and K. Tuominen, JHEP 0403, 044 (2004) [arXiv:hep-ph/0306069]; A. Mocsy, F. Sannino and K. Tuominen, Phys. Rev. Lett. 91, 092004 (2003) [arXiv:hep-ph/0301229].

[2] F. Sannino and K. Tuominen, Phys. Rev. D 70, 034019 (2004) [arXiv:hep-ph/0403175].

[3] K. Fukushima, Phys. Lett. B 591, 277 (2004) [arXiv:hep-ph/0310121]; K. Fukushima, Phys. Rev. D 68, 045004 (2003) [arXiv:hep-ph/0303225]; K. Fukushima, Phys. Lett. B 553, 38 (2003) [arXiv:hep-ph/0209311].

[4] C. Ratti, M. A. Thaler and W. Weise, Phys. Rev. D 73, 014019 (2006) [arXiv:hep-ph/0506234];

C. Ratti, S. Roessner and W. Weise, Phys. Lett. B 649 (2007) 57 [arXiv:hep-ph/0701091].

[5] S. Roessner, T. Hell, C. Ratti and W. Weise, Nucl. Phys. A 814 (2008) 118 [arXiv:0712.3152 [hep-ph]].

[6] M. Ciminale, G. Nardulli, M. Ruggieri and R. Gatto, Phys. Lett. B 657 (2007) 64 [arXiv:0706.4215 [hep-ph]]. 
[7] P. Costa, M. C. Ruivo and C. A. de Sousa, arXiv:0801.3417 [hep-ph], P. Costa, C. A. de Sousa, M. C. Ruivo and H. Hansen, arXiv:0801.3616 [hep-ph].

[8] T. Kahara and K. Tuominen, Phys. Rev. D 80, 114022 (2009) [arXiv:0906.0890 [hep-ph]].

[9] B. J. Schaefer, J. M. Pawlowski and J. Wambach, Phys. Rev. D 76 (2007) 074023 [arXiv:0704.3234 [hep-ph]].

[10] T. Kahara and K. Tuominen, Phys. Rev. D 78 (2008) 034015 [arXiv:0803.2598 [hep-ph]].

[11] V. Skokov, B. Friman, E. Nakano, K. Redlich and B. J. Schaefer, arXiv:1005.3166 [hep-ph].

[12] V. Skokov, B. Friman and K. Redlich, arXiv:1008.4570 [hep-ph].

[13] A. Mocsy, I. N. Mishustin and P. J. Ellis, Phys. Rev. C 70 (2004) 015204 [arXiv:nucl-th/0402070].

[14] E. Nakano, B. J. Schaefer, B. Stokic, B. Friman and K. Redlich, Phys. Lett. B 682 (2010) 401 [arXiv:0907.1344 [hep-ph]].

[15] G. Marko and Z. Szep, arXiv:1006.0212 [hep-ph].

[16] T. Kahara and K. Tuominen, arXiv:1006.3931 [hep-ph].

[17] S. Roessner, C. Ratti and W. Weise, Phys. Rev. D 75 (2007) 034007 [arXiv:hep-ph/0609281].

[18] B. J. Schaefer and M. Wagner, Phys. Rev. D 79 (2009) 014018 [arXiv:0808.1491 [hep-ph]].

[19] L. Ferroni, V. Koch and M. B. Pinto, arXiv:1007.4721 [nucl-th].

[20] Y. Hatta and T. Ikeda, Phys. Rev. D 67 (2003) 014028 [arXiv:hep-ph/0210284].

[21] E. S. Bowman and J. I. Kapusta, Phys. Rev. C 79, 015202 (2009) [arXiv:0810.0042 [nucl-th]].

[22] Y. Aoki, Z. Fodor, S. D. Katz and K. K. Szabo, Phys. Lett. B 643 (2006) 46 [arXiv:hep-lat/0609068]. 